
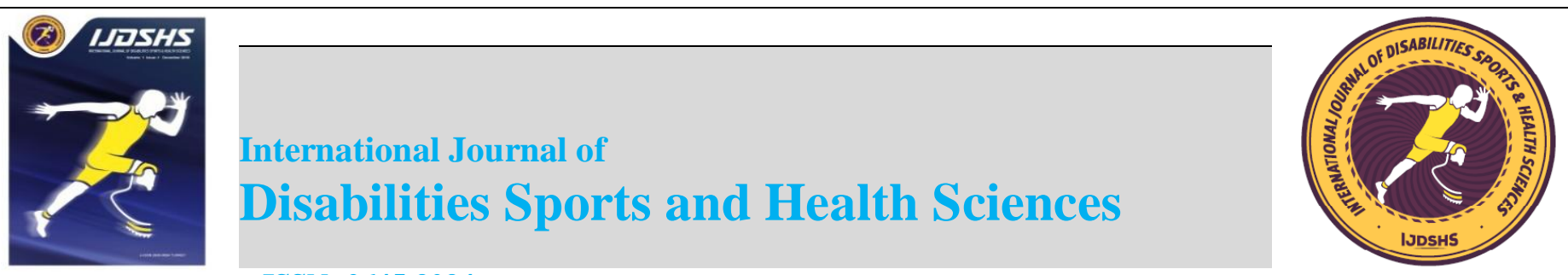

\title{
The Relationship Between Caregiver Workload and Stress Levels with Clinical Symptom Severity in Cerebral Palsy
}

\author{
Fatih ÖZDEN*1 ${ }^{\text {iD }}$, Ahmet Furkan ARIK $^{2}$, Mehmet ÖZKESKIN ${ }^{3}$ iD and Serkan BAKIRHAN ${ }^{\text {(DD }}$ \\ ${ }^{* 1}$ Muğla Sitkı Koçman University, Köyceğiz Vocational School of Health Services, Elderly Care Department, Muğla, Turkey \\ ${ }^{2}$ Marmara University, Institute of Health Sciences, Department of Physiotherapy, İstanbul, Turkey \\ ${ }^{3,4,}$ Ege University, Faculty of Health Sciences, Physical Therapy and Rehabilitation Department, İzmir, Turkey \\ *Corresponding author: fatihozden@mu.edu.tr
}

\begin{abstract}
The aim of this study is to compare the functional status, spasticity, motor function status of children with cerebral palsy and their caregivers' workload and stress levels. A single-center prospective cross-sectional study was carried out with 30 children with Cerebral Palsy (CP) and their 30 caregivers. The clinical status of the children was assessed with the Modified Ashworth Scale (MAS), Gross Motor Function Classification System (GMFCS), The Functional Independence Measure for Children (WeeFIM). The caregivers' workload and stress levels were evaluated with Bakas Caregiving Outcomes Scale (BCOS) and Perceived Stress Scale (PSS), respectively. The mean age of the children was $11.46 \pm 7.45$ years. In correlation analysis, the caregivers' BCOS score was related to the children's MAS score $(\mathrm{p}<0.05)$. On the other hand, there was a significant relationship between BCOS and PSS scores of the caregivers $(\mathrm{p}<0.05)$. Greater levels of spasticity in the children were associated with greater levels of workload. Stress level is higher in caregivers with a high workload.
\end{abstract}

Keywords

Cerebral Palsy, Caregiver; Workload; Stress, Clinical Stiuation

\section{INTRODUCTION}

Cerebral palsy (CP) is defined as a group of motor syndromes that occur as a result of immature non-progressive pathologies in the brain and are mostly accompanied by different neurodevelopmental disorders (Krigger, 2006; P. Rosenbaum et al., 2007; Sankar \& Mundkur, 2005). CP is the most common neuromotor disorder and the main cause of functional impairment in childhood (Fernández-Alcántara et al., 2015). The prevalence of CP in children between 2-16 years of age is higher in Turkey from developed countries and was reported to be 0.44\% (Serdaroglu, Cansu, Md, \& Tezcan, 2006). The clinical situation and the severity of various symptoms in children vary over time. For instance, initial hypotonia may be replaced by spasticity or involuntary movements later on. Movement and posture disorders in children with cerebral palsy constitute the basic clinical picture, but mental retardation, seizures, eye problems (such as homonymous hemianopia, strabismus), stereognosis, proprioception disorders, and hearing disorders may accompany the clinical picture. In this case, the rehabilitation process is more difficult and it is difficult to gain physical independence (Serdaroglu et al., 2006).

$\mathrm{CP}$ is a chronic and complex condition that requires high maintenance costs and causes serious impairments in the general motor skills of the children. Spastic paralysis causes various problems such as mental retardation, mental impairment, speech impairment, vision-hearing impairment, 
and limitations in self-care functions such as feeding, dressing, bathing, and ambulation. All of these have negative effects on children's daily life activities and parents' family dynamics. Mothers of children with $\mathrm{CP}$ have important roles in the treatment and management of CP (El, Peker, Bozan, Berk, \& Koşay, 2007). In particular, functional disorders cause essential problems. The functional state is "the ability of a person to participate in society without physical and mental restrictions".

Caring is a natural role of mothers; however, caring for a child with functional limitations and long-term addiction is completely different and affects the physical and emotional functions of mothers (Garip et al., 2017). Families play an important role in providing care to children with $\mathrm{CP}$. Caregivers often become isolated from preexisting relationships and social activities because of their responsibilities and roles. Especially the physical and psychological complications of the disease and intensive treatment sessions severely burden caregivers' life. One of the most important factors that increase caregiving work is the deterioration of the child's physical abilities. It was observed that the functional capacity decreased in the advanced stages of the disease when the motor symptoms of the disease were exacerbated, and it was also shown that caregiver responsibilities and workload increased during this period (Stetz \& Brown, 1997).

Chronic emotional stress in families; increases their anxiety and stress levels, as well as causes overcoming the workload more difficult. Feelings of social isolation and loneliness can be experienced. This situation decreases life satisfaction and quality of life (Deniz, Dilmaç, \& Arıcak, 2009). In this study, the level of the child's clinical condition will be correlated with the caregiver's workload and anxiety. In this way, it will be revealed whether the workload and anxiety situations of the caregiver are related to the clinical situation. The aim of this study is to compare the functional status, spasticity, motor function status of children with cerebral palsy and their caregivers' workload and stress levels.

\section{MATERIALS AND METHODS}

\section{Study Design and Participants}

A single-center prospective cross-sectional study was carried out with 30 children with CP and their 30 caregivers in " $\mathrm{X}$ " Special Education and Rehabilitation Center. The inclusion criteria were; (1) children with $\mathrm{CP}$, (2) aged $<18$ years. The exclusion criteria of the study were; (1) cognitive problems of the mother who unable to fill the questionnaire, (2) mothers' caregiving to another chronic patient, (3) a history of chronic disease in mothers, (4) mothers caring for a child <2 years, (5) mothers who are pregnant, (6) caregivers who do not give consent to participate in the study. The study protocol was approved by the ethics committee of Ege University (No: 20-10.1T/22). All of the assessments were conducted in accordance with the Helsinki declaration. All cases provided a written informed consent approved by the ethics committee.

\section{Data Collection}

The children and their caregivers were evaluated on the same day of inclusion. All muscle strength measurements and other performance tests were conducted by the same researcher (physiotherapist). Demographic, physical, and pathological information of the children with $\mathrm{CP}$ were recorded. Also, the demographical characteristics of the caregivers were obtained in a face-to-face interview. Children and their caregivers were assessed using the outcome measurements listed below.

\section{Modified Ashworth Scale (MAS)}

MAS was used to evaluate muscle tone of the children. In the evaluation, scores between 0-4 are given according to the increase in the amount of resistance felt during passive movement of the arm and leg $(0=$ normal muscle tone, $1=$ slight increase in tonus felt at the end of the joint range of motion (ROM), $1+=$ slight tone felt in less than half of the ROM. 2 = significant increase in a tone that does not prevent passive movement but is felt in most of ROM, 3 = significant increase in a tone that makes passive movement difficult during ROM, $4=$ severe increase in tone, affected movement which is in a rigid state (Charalambous, 2014; Mutlu, Livanelioglu, \& Gunel, 2008).

\section{Gross Motor Function Classification System (GMFCS)}

GMFCS was used to classify the severity of the impairment in children's gross motor functions. It is a valid and reliable standardized system that classifies the gross motor functions of children with CP between the ages of 0-12 in five levels. Children with cerebral palsy are the least dependent on level 1 and the most dependent on 
level 5 in motor functions (Palisano, Rosenbaum, Bartlett, \& Livingston, 2008; P. L. Rosenbaum, Palisano, Bartlett, Galuppi, \& Russell, 2008).

\section{The Functional Independence Measure for Children (WeeFIM)}

Turkish version of the WeeFIM was used for the functional status assessment of children with cerebral palsy. WeeFIM is adapted from the Functional Independence Measure (FIM) for adults and can be applied by direct observation, interview, or a combination of both. It consists of six sub-parameters and 18 questions. Subparameters: self-care (6 items), sphincter control (2 items), transfer (3 items), locomotion (3 items), communication (2 items), and social status (3 items). Scoring is done between 1 (fully dependent) and 7 (fully independent) points. The lowest possible score is 18 and the highest score is 126 (Msall et al., 1994; Tur et al., 2009).

\section{Bakas Caregiving Outcomes Scale (BCOS)}

Turkish version of BCOS was used for the workload assessment of caregivers. The scale shows both positive and negative effects. The scale consists of 15 questions scored from +3 (the best direction) to -3 (the worst direction). It is a Likert type scale ranging from 1 to 7 . A minimum of 15 and a maximum of 105 points can be obtained on the scale. As the score increases, it is interpreted as "change in the good direction" and as the score decreases it is interpreted as "change in the bad direction" (Bakas \& Champion, 1999; Kavlak et al., 2018).

\section{Perceived Stress Scale (PSS)}

Consisting of 10 items, the PSS is designed to measure how stressful certain situations are perceived in a person's life. Participants evaluate each item on a 5-point Likert-type scale ranging from "Never (0)" to "Very often (4)". Items containing positive statements are scored in reverse. PSS is scored between 0 and 40. High score indicates that the person has an excessive perception of stress. The Turkish version of PSS was used in our study (Eskin, Harlak, Demirkıran, \& Dereboy; Lee, 2012).

\section{Statistical Analysis}

The statistical package for the social sciences for Windows version 25 (SPSS Inc, Chicago, IL) computer program was used for data analysis. Quantitative variables were presented as mean \pm standard deviation (X $\pm \mathrm{SD}$ ), range (minimummaximum), and qualitative variables percent (\%).
Results were considered statistically significant when $\mathrm{p}$-value $<0.05$. The Shapiro-Wilk tests were used to determine the normal distribution. The "Spearman rank correlation coefficient" was used to investigate the relationship between caregiver workload and stress levels of the caregivers with clinical symptom severity. The Pearson correlation coefficients were interpreted as; $0-0.19=$ very low, $0.20-0.39=$ low, $0.40-0.69=$ medium, $0.70-0.89=$ high, $0.90-1.0=$ very high (Streiner, Norman, \& Cairney, 2015).

\section{RESULTS}

The mean age of the children was 11.46 \pm 7.45 years. More than half of children was male (\%80). Body Mass Index of the children was $19.95 \pm 5.49 \mathrm{~kg} / \mathrm{m}^{2}$ (classified as normal). Less than half of the children use assistive devices or orthoses $(43.3 \%)$. The mean values of the children's physical and pathological status were presented in Table $1.90 \%$ of the caregivers were urban residents.

Table 1: Physical and pathological characteristics of the children

\begin{tabular}{lc}
\hline & Total $(\mathbf{n}=\mathbf{3 0})$ \\
\hline Age $($ years, mean \pm SD) & $11.46 \pm 7.45$ \\
Height $(\mathrm{m}$, mean \pm SD) & $1.33 \pm 0.32$ \\
Weight $(\mathrm{kg}$, mean \pm SD) & $40.31 \pm 23.91$ \\
BMI $(\mathrm{kg} / \mathrm{m} 2$, mean \pm SD) & $19.95 \pm 5.49$ \\
Assistive Device & \\
Yes $(\mathrm{n}, \%)$ & $13(43.3)$ \\
No $(\mathrm{n}, \%)$ & $17(56.7)$ \\
Modified Ashworth $($ mean \pm SD) & $2.80 \pm 1.51$ \\
GMFCS (mean \pm SD) & $3.06 \pm 1.08$ \\
WeeFIM (mean \pm SD) & $68.7 \pm 34.2$ \\
\hline
\end{tabular}

SD: standard deviation, n: number of patients, \%: percentage, GMFCS: Gross Motor Function Classification System, WeeFIM: The Functional Independence Measure for Children

The socio-demographic characteristics of the caregivers and the absolute values of stress and workload scores are given in Table 2. In correlation analysis, the BCOS score of the caregivers was related with MAS score of the children $(\mathrm{p}<0.05)$ (Table 3). On the other hand, there were significant relationship between BCOS and PSS scores of the caregivers $(\mathrm{p}<0.05)$. Also, GMFC and WeeFIM scores of the children were strongly correlated $(\mathrm{p}<0.001)$. 
Table 2: Absolute values (standard deviation, min-max) for the caregivers

\begin{tabular}{lc}
\hline $\mathbf{n : 3 0}$ & Mean \pm SD \\
\hline Marital status (n, \%) & $29(96.7)$ \\
Married & $1(3.3)$ \\
Single & $27(90)$ \\
Residence (n, \%) & $3(10)$ \\
Urban & \\
Rural & $11(39,9)$ \\
Education (n, \%) & $10(33.3)$ \\
Literate & $5(16.6)$ \\
Primary school & $4(13.3)$ \\
High school & $10.56 \pm 7.23$ \\
University or postgraduate & $17.20 \pm 4.58$ \\
Caregiving duration (mean \pm SD) & $51.56 \pm 10.98$ \\
PSS (mean \pm SD) & \\
BCOS (mean \pm SD) & \\
\hline SD: standard deviation, n: number of patients, \%: percentage, PSS: Perceived Stress Scale, BCOS: Bakas Caregiving & \\
Outcomes Scale &
\end{tabular}

Table 3: Correlation between Modified Ashworth, GMFCS, WeeFIM with PSS and BCOS

\begin{tabular}{lcc}
\hline n:30 & PSS & BCOS \\
\hline Modified Ashworth & 0.80 & $-0.37^{*}$ \\
GMFCS & -0.29 & 0.14 \\
WeeFIM & 0.05 & 0.11 \\
\hline
\end{tabular}

*: $\mathrm{p}<0.05, * *: \mathrm{p}<0.01$, PSS: Perceived Stress Scale, BCOS: Bakas Caregiving Outcomes Scale, GMFCS: Gross Motor

Function Classification System, WeeFIM: The Functional Independence Measure for Children

\section{DISCUSSION}

The present study aimed to demonstrate the relationship between the functional status, spasticity, motor function status of children with cerebral palsy and their caregivers' workload and stress levels. According to the results of the study we concluded that, greater levels of spasticity in the children were associated with greater levels of workload. In addition, the workload of caregivers was associated with stress levels. Stress level was found to be higher in the caregivers with high workload. However, there was no relationship between stress level of the caregivers and clinical parameters of the children.

Spasticity is one of the primary factors affecting the functional level and independence of children with cerebral palsy (Liao, Jeny, Lai, Cheng, \& Hu, 1997; Ross \& Engsberg, 2007). The increase in spasticity causes children to be unable to perform daily living activities independently (Park, 2018; Shamsoddini, Amirsalari, Hollisaz, Rahimnia, \& Khatibi-Aghda, 2014). In this case, the caregivers' workload will increase and they will make more effort for the child to perform daily life activities (Siritaratiwat, Inthachom, \& Warnset, 2012). We used MAS, one of the most common and essential assessment tools used in evaluating spasticity, and determined the children's spasticity levels ( $\mathrm{Li}, \mathrm{Wu}, \& \mathrm{Li}, 2014)$. On the other hand, BCOS was one of the most practical tools used to assess caregivers' workload (Bakas, Champion, Perkins, Farran, \& Williams, 2006). As expected, the workload and level of spasticity were correlated. However, there was no relationship between other clinical parameters and BCOS. This situation may be due to our relatively small sample size. Our study was carried out with a pragmatic approach, using the conditions and facilities available. Among the clinical parameters, it can be claimed that spasticity is the parameter that provokes the most workload. However, more fundamental analysis can be obtained with a larger number of samples and a model to be created using multifactorial variables in regression analysis. Our study may be an essential initial finding to consider spasticity as an indicator of workload in future studies.

A recent study by Eminel et al. concluded that caregivers of children with walking disabilities had higher workloads (Eminel, Kahraman, \& Genc, 2020). They also stated that 
these caregivers have more physical and psychological problems. The results obtained from the study are consistent with our research. Similarly, another recent study examined stress levels in families and caregivers of children with cerebral palsy (Fritz \& Sewell-Roberts, 2020). It has been demonstrated that the children's clinical condition is not consequently linked to the stress level of the family. Since this study is qualitative research, it was stated that other factors that may cause stress levels may also be significant at the family's psychological level. It has been described that the social, behavioral, and physiological characteristics of the child and the financial and socioeconomic level of the family can be determining factors in terms of stress level.

In another study, different results were obtained in terms of different psychological parameters between mothers' anxiety levels and the clinical condition of individuals with cerebral palsy (Akmeşe, Mutlu, \& Günel, 2007). In our study, the caregiver's stress level was not found to be related to the child's clinical condition. All of the caregivers were the families of the children. We recorded the socio-demographic status of the families. However, all of these parameters were categorical variables. It could not be examined in detail whether there is a difference in terms of stress and workload among individuals with different education levels and economic income. For this reason, the relatively small sample size is the most significant limitation of our study. Besides, the stress level could be evaluated by including different sub-parameters. In this way, it can be seen in what sub-dimensions of stress there is an increase in children's caregivers. Last but not least, forming the sample homogeneously from individuals with different cultural segments and economic income levels can yield more precise results.

\section{Conclusion}

Greater levels of spasticity in the children were associated with greater levels of workload. Besides, the workload of caregivers was associated with stress levels. Stress level is higher in the caregivers with high workload.

\section{Ethical approval}

The study was carried out in accordance with the ethical principles and the Helsinki Declaration. Informed consent of the patients was obtained. The study protocol was approved by the ethics committee of Ege University (No:20-10.1T/22)

\section{Declaration of Interest Statement}

The authors report no conflicts of interest and certify that no funding has been received for this study and/or preparation of this manuscript.

\section{REFERENCES}

Akmeşe, P. P., Mutlu, A., \& Günel, M. K. (2007). Investigation of the anxiety level of mothers of children with cerebral paralysis. Journal of Child Health and Diseases, 50, 236-240.

Bakas, T., \& Champion, V. (1999). Development and psychometric testing of the Bakas Caregiving Outcomes Scale. Nursing Research, 48(5), 250-259.

Bakas, T., Champion, V., Perkins, S. M., Farran, C. J., \& Williams, L. S. (2006). Psychometric testing of the revised 15-item Bakas Caregiving Outcomes Scale. Nursing Research, 55(5), 346-355.

Charalambous, C. P. (2014). Interrater reliability of a modified Ashworth scale of muscle spasticity. In Classic papers in orthopaedics (pp. 415-417): Springer.

Deniz, M. E., Dilmaç, B., \& Arıcak, O. T. (2009). Examination of state-trait anxiety and life satisfaction of parents with disabled children. International Journal of Human Sciences, 6(1), 953-968.

El, Ö., Peker, Ö., Bozan, Ö., Berk, H., \& Koşay, C. (2007). General characteristics of cerebral palsy patients. Dokuz Eylul University Faculty of Medicine Journal, 21(2), 75-80.

Eminel, A. G., Kahraman, T., \& Genc, A. (2020). Physical workload during caregiving activities and related factors among the caregivers of children with cerebral palsy. Irish Journal of Medical Science (1971-), 19.

Eskin, M., Harlak, H., Demirkıran, F., \& Dereboy, Ç. (2013). Adaptation of the perceived stress scale to Turkish: reliability and validity analysis.

Fernández-Alcántara, M., García-Caro, M. P., Laynez-Rubio, C., Pérez-Marfil, M. N., Martí-García, C., Benítez-Feliponi, Á., . . . Cruz-Quintana, F. (2015). Feelings of loss in parents of children with infantile cerebral 
palsy. Disability and health journal, 8(1), 93-101.

Fritz, H., \& Sewell-Roberts, C. (2020). Family stress associated with cerebral palsy. Cerebral palsy, 515-545.

Garip, Y., Ozel, S., Tuncer, O. B., Kilinc, G., Seckin, F., \& Arasil, T. (2017). Fatigue in the mothers of children with cerebral palsy. Disability and rehabilitation, 39(8), 757762.

Kavlak, E., Kara, G., Tekin, F., Altuğ, F., Ök, N., \& Hande, Ş. (2018). Stress and depression levels of mothers who give care to children with cerebral palsy and mental retardation: a comparison study.

Krigger, K. W. (2006). Cerebral palsy: an overview. American family physician, 73(1), 91-100.

Lee, E.-H. (2012). Review of the psychometric evidence of the perceived stress scale. Asian nursing research, 6(4), 121-127.

Li, F., Wu, Y., \& Li, X. (2014). Test-retest reliability and inter-rater reliability of the Modified Tardieu Scale and the Modified Ashworth Scale in hemiplegic patients with stroke. Eur J Phys Rehabil Med, 50(1), 9-15.

Liao, H. F., Jeny, S. F., Lai, J. S., Cheng, C. K., \& $\mathrm{Hu}$, M. H. (1997). The relation between standing balance and walking function in children with spastic diplegic cerebral palsy. Developmental Medicine \& Child Neurology, 39(2), 106-112.

Msall, M. E., DiGaudio, K., Duffy, L. C., LaForest, S., Braun, S., \& Granger, C. V. (1994). WeeFIM: normative sample of an instrument for tracking functional independence in children. Clinical pediatrics, 33(7), 431-438.

Mutlu, A., Livanelioglu, A., \& Gunel, M. K. (2008). Reliability of Ashworth and Modified Ashworth scales in children with spastic cerebral palsy. BMC musculoskeletal disorders, 9(1), 44.

Palisano, R. J., Rosenbaum, P., Bartlett, D., \& Livingston, M. H. (2008). Content validity of the expanded and revised Gross Motor Function Classification System. Developmental Medicine \& Child Neurology, 50(10), 744-750.
Park, E.-Y. (2018). Path analysis of strength, spasticity, gross motor function, and healthrelated quality of life in children with spastic cerebral palsy. Health and quality of life outcomes, 16(1), 1-7.

Rosenbaum, P., Paneth, N., Leviton, A., Goldstein, M., Bax, M., Damiano, D., . . Jacobsson, B. (2007). A report: the definition and classification of cerebral palsy April 2006. Dev Med Child Neurol Suppl, 109(suppl 109), 8-14.

Rosenbaum, P. L., Palisano, R. J., Bartlett, D. J., Galuppi, B. E., \& Russell, D. J. (2008). Development of the gross motor function classification system for cerebral palsy. Developmental Medicine \& Child Neurology, 50(4), 249-253.

Ross, S. A., \& Engsberg, J. R. (2007). Relationships between spasticity, strength, gait, and the GMFM-66 in persons with spastic diplegia cerebral palsy. Archives of physical medicine and rehabilitation, 88(9), 1114-1120.

Sankar, C., \& Mundkur, N. (2005). Cerebral palsydefinition, classification, etiology and early diagnosis. The Indian Journal of Pediatrics, 72(10), 865-868.

Serdaroglu, A., Cansu, A., Md, S. Ö., \& Tezcan, S. (2006). Prevalence of cerebral palsy in Turkish children between the ages of 2 and 16 years. Developmental Medicine \& Child Neurology, 48(6), 413-416.

Shamsoddini, A., Amirsalari, S., Hollisaz, M.-T., Rahimnia, A., \& Khatibi-Aghda, A. (2014). Management of spasticity in children with cerebral palsy. Iranian journal of pediatrics, 24(4), 345.

Siritaratiwat, W., Inthachom, R., \& Warnset, S. (2012). The usage of a hand-made chair at home for children with moderate to severe cerebral palsy: preliminary study. Journal of the Medical Association of Thailand= Chotmaihet thangphaet, 95(2), 198.

Stetz, K. M., \& Brown, M. A. (1997). Taking care: caregiving to persons with cancer and AIDS. Cancer Nursing, 20(1), 12-22.

Streiner, D. L., Norman, G. R., \& Cairney, J. (2015). Health measurement scales: a practical guide to their development and use: Oxford University Press, USA. 
Tur, B. S., Küçükdeveci, A. A., Kutlay, Ş., Yavuzer, G., Elhan, A. H., \& Tennant, A. (2009). Psychometric properties of the WeeFIM in children with cerebral palsy in Turkey. Developmental Medicine \& Child Neurology, 51(9), 732-738.

How to cite this article: Özden, F., Arık, A.F., Özkeskin, M. and Bakırhan, S. (2021). The Relationship Between Caregiver Workload and Stress Levels with Clinical Symptom Severity in Cerebral Palsy. Int J Disabil Sports Health Sci;4(1):38-44. https://doi.org/10.33438/ijdshs. 833840 